\title{
Evaluation and Optimization of APGC Parameters for Analysis of Selected Hop Essential Oil Volatiles
}

Laura Knoke, Nils Rettberg*

Research Institute for Beer and Beverage Analysis, Versuchs- und Lehranstalt für Brauerei in Berlin (VLB) e.V., Seestr. 13, Berlin, 13353 Germany

\section{SUPPORTING INFORMATION}

Table S1. Formula and $\mathrm{m} / \mathrm{z}$ of the molecular ions $\left(\left[\mathrm{M}^{*}\right]^{+}\right)$, protonated molecules $\left([\mathrm{M}+\mathrm{H}]^{+}\right)$and fragment ions monitored during optimization, and characteristic ions and their formation under final APGC method parameters for all analytes.

\begin{tabular}{|c|c|c|c|c|c|c|c|}
\hline \multirow{2}{*}{ analyte } & \multirow{2}{*}{ formula } & \multicolumn{4}{|c|}{$\mathrm{m} / \mathrm{z}$ (theoretical) } & \multicolumn{2}{|c|}{ characteristic ion } \\
\hline & & {$\left[\mathrm{M}^{\bullet}\right]^{+}$} & {$[\mathrm{M}+\mathrm{H}]^{+}$} & $\mathrm{F} 80^{\mathrm{a}}$ & $\mathrm{F} 200^{\mathrm{a}}$ & $\begin{array}{c}\mathrm{m} / \mathrm{z} \\
\text { (theoretical) }\end{array}$ & ion \\
\hline myrcene & $\mathrm{C}_{10} \mathrm{H}_{16}$ & 136.1247 & 137.1325 & 91.0548 & 81.0704 & 137.1325 & $\mathrm{M}+\mathrm{H}$ \\
\hline (-)- $\beta$-pinene & $\mathrm{C}_{10} \mathrm{H}_{16}$ & 136.1247 & 137.1325 & 91.0548 & 81.0704 & 137.1325 & $\mathrm{M}+\mathrm{H}$ \\
\hline$(+)$-limonene & $\mathrm{C}_{10} \mathrm{H}_{16}$ & 136.1247 & 137.1325 & 91.0548 & 81.0704 & 137.1325 & $\mathrm{M}+\mathrm{H}$ \\
\hline$\alpha$-humulene & $\mathrm{C}_{15} \mathrm{H}_{24}$ & 204.1873 & 205.1951 & 91.0548 & 149.1330 & 205.1951 & $\mathrm{M}+\mathrm{H}$ \\
\hline$\beta$-caryophyllene & $\mathrm{C}_{15} \mathrm{H}_{24}$ & 204.1873 & 205.1951 & 91.0548 & 149.1330 & 205.1951 & $\mathrm{M}+\mathrm{H}$ \\
\hline 2-undecanone & $\mathrm{C}_{11} \mathrm{H}_{22} \mathrm{O}$ & 170.1665 & 171.1743 & 95.0861 & 97.1017 & 171.1743 & $\mathrm{M}+\mathrm{H}$ \\
\hline linalool & $\mathrm{C}_{10} \mathrm{H}_{18} \mathrm{O}$ & 154.1352 & 155.1430 & 91.0548 & 137.1325 & 137.1325 & $\mathrm{M}-\mathrm{OH}$ \\
\hline$(+)$ - $\alpha$-terpineol & $\mathrm{C}_{10} \mathrm{H}_{18} \mathrm{O}$ & 154.1352 & 155.1430 & 91.0548 & 137.1325 & 137.1325 & $\mathrm{M}-\mathrm{OH}$ \\
\hline cis-linalooloxide & $\mathrm{C}_{10} \mathrm{H}_{18} \mathrm{O}_{2}$ & 170.1301 & 171.1380 & 91.0548 & 153.1274 & 153.1274 & $\mathrm{M}-\mathrm{OH}$ \\
\hline (-)-caryophyllenoxide & $\mathrm{C}_{15} \mathrm{H}_{24} \mathrm{O}$ & 220.1822 & 221.1900 & 91.0548 & 203.1800 & 203.1800 & $\mathrm{M}-\mathrm{OH}$ \\
\hline 2-methylbutyl isobutyrate & $\mathrm{C}_{9} \mathrm{H}_{18} \mathrm{O}_{2}$ & 158.1301 & 159.1380 & \multicolumn{2}{|c|}{89.0580} & 89.0580 & $\mathrm{M}-\mathrm{C}_{5} \mathrm{H}_{9}$ \\
\hline 3-methylbutyl isobutyrate & $\mathrm{C}_{9} \mathrm{H}_{18} \mathrm{O}_{2}$ & 158.1301 & 159.1380 & 115.0758 & 89.0580 & 89.0580 & $\mathrm{M}-\mathrm{C}_{5} \mathrm{H}_{9}$ \\
\hline isobutyl isobutyrate & $\mathrm{C}_{8} \mathrm{H}_{16} \mathrm{O}_{2}$ & 144.1145 & 145.1223 & \multicolumn{2}{|c|}{89.0580} & 89.0580 & $\mathrm{M}-\mathrm{C}_{4} \mathrm{H}_{7}$ \\
\hline butyl isobutyrate & $\mathrm{C}_{8} \mathrm{H}_{16} \mathrm{O}_{2}$ & 144.1145 & 145.1223 & \multicolumn{2}{|c|}{89.0580} & 89.0580 & $\mathrm{M}-\mathrm{C}_{4} \mathrm{H}_{7}$ \\
\hline 2-methylbutyl 2-methylbutyrate & $\mathrm{C}_{10} \mathrm{H}_{20} \mathrm{O}_{2}$ & 172.1458 & 173.1536 & \multicolumn{2}{|c|}{103.0761} & 103.0761 & $\mathrm{M}-\mathrm{C}_{5} \mathrm{H}_{9}$ \\
\hline 2-methylbutyl 3-methylbutyrate & $\mathrm{C}_{10} \mathrm{H}_{20} \mathrm{O}_{2}$ & 172.1458 & 173.1536 & \multicolumn{2}{|c|}{103.0761} & 103.0761 & $\mathrm{M}-\mathrm{C}_{5} \mathrm{H}_{9}$ \\
\hline propyl 2-methylbutyrate & $\mathrm{C}_{8} \mathrm{H}_{16} \mathrm{O}_{2}$ & 144.1145 & 145.1223 & \multicolumn{2}{|c|}{103.0761} & 103.0761 & $\mathrm{M}-\mathrm{C}_{3} \mathrm{H}_{5}$ \\
\hline methyl 2-methylbutyrate & $\mathrm{C}_{6} \mathrm{H}_{12} \mathrm{O}_{2}$ & 116.0832 & 117.0910 & 101.0602 & 85.0653 & 117.0910 & $\mathrm{M}+\mathrm{H}$ \\
\hline methyl geranate & $\mathrm{C}_{11} \mathrm{H}_{18} \mathrm{O}_{2}$ & 182.1301 & 183.1380 & \multicolumn{2}{|c|}{123.1174} & 123.1174 & $\mathrm{M}-\mathrm{C}_{2} \mathrm{H}_{3} \mathrm{O}_{2}$ \\
\hline geranyl acetate & $\mathrm{C}_{12} \mathrm{H}_{20} \mathrm{O}_{2}$ & 196.1458 & 197.1536 & 91.0548 & 137.1325 & 137.1330 & $\mathrm{M}-\mathrm{C}_{2} \mathrm{H}_{3} \mathrm{O}_{2}$ \\
\hline geranyl propionate & $\mathrm{C}_{13} \mathrm{H}_{22} \mathrm{O}_{2}$ & 210.1614 & 211.1693 & 91.0548 & 137.1325 & 137.1330 & $\mathrm{M}-\mathrm{C}_{3} \mathrm{H}_{5} \mathrm{O}_{2}$ \\
\hline geranyl isobutyrate & $\mathrm{C}_{14} \mathrm{H}_{24} \mathrm{O}_{2}$ & 224.1771 & 225.1849 & 91.0548 & 137.1325 & 137.1330 & $\mathrm{M}-\mathrm{C}_{4} \mathrm{H}_{7} \mathrm{O}_{2}$ \\
\hline
\end{tabular}

a: F80 and F200 = fragment ions at cone gas flows of 80 and $200 \mathrm{~L} / \mathrm{h}$, respectively. 


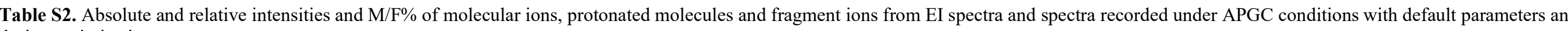
during optimization.

\begin{tabular}{|c|c|c|c|c|c|c|c|c|c|c|c|c|c|c|c|c|}
\hline \multirow{3}{*}{ analyte } & & \multirow{2}{*}{\multicolumn{3}{|c|}{ E (NIST) }} & \multirow{2}{*}{\multicolumn{12}{|c|}{ optimization of cone gas $[\mathrm{L} / \mathrm{h}]$}} \\
\hline & & & & & \multicolumn{5}{|c|}{ default parameters } & & & & & & \multicolumn{2}{|c|}{$\begin{array}{c}\text { optimization of cone gas }[\mathrm{L} / \mathrm{h}] \\
80\end{array}$} \\
\hline & & {$\left[\mathbf{M}^{i}\right]^{+}$} & {$[\mathbf{M}+\mathbf{H}]^{+}$} & $\mathbf{F}$ & {$\left[\mathbf{M i}^{\mathbf{+}}\right]^{+}$} & {$[\mathbf{M}+\mathbf{H}]^{+}$} & F80 & F200 & $\left|M^{0}\right|^{+}$ & {$[\mathbf{M}+\mathbf{H}]^{+}$} & F80 & F200 & {$\left[\mathbf{M i}^{+}\right]^{+}$} & {$\left[\mathbf{M}+\mathbf{H}^{+}\right.$} & $\begin{array}{ll}\text { F80 } \\
\end{array}$ & F200 \\
\hline \multirow{3}{*}{ myrcene } & intensity abs. & & & 10000 & 1082623 & 2062251 & 1089806 & 8529565 & 203272 & 7488882 & 4289143 & 1432670 & 25938 & 2153470 & 1248756 & 271228 \\
\hline & intensity & 4.1 & 0.5 & 100.0 & 12.7 & 24.2 & 12.8 & 100.0 & 2.7 & 100.0 & 57.3 & 19.1 & 1.2 & 100.0 & 58.0 & 12.6 \\
\hline & M/F\% & 4.1 & 0.5 & & $\begin{array}{r}12.7 \\
1072198\end{array}$ & 24.2 & & & $\begin{array}{l}4.7 \\
\end{array}$ & $\begin{array}{r}174.6 \\
01019079\end{array}$ & & & 2.1 & 172.4 & & \\
\hline \multirow[b]{2}{*}{$(-)-\beta-p i n e n e$} & $\begin{array}{l}\text { intensity abs. } \\
\text { intensity \% }\end{array}$ & $\begin{array}{l}731 \\
73.3\end{array}$ & 100 & $\begin{array}{l}10000 \\
100\end{array}$ & $\begin{aligned} 1972448 \\
168\end{aligned}$ & $\begin{array}{l}2637210 \\
225\end{array}$ & 1353426 & 11724633 & 266771 & 10645978 & 5817723 & 1774951 & 24827 & 2894873 & 1727870 & 261227 \\
\hline & $\begin{array}{l}\text { intensity } \\
\text { MFF\% }\end{array}$ & $\begin{array}{l}7.3 \\
7.3\end{array}$ & $\begin{array}{l}1.0 \\
1.0\end{array}$ & 100.0 & $\begin{array}{l}16.8 \\
16.8\end{array}$ & $\begin{array}{l}22.5 \\
22.5\end{array}$ & 11.5 & 100.0 & $\begin{array}{l}2.5 \\
4.6\end{array}$ & $\begin{array}{l}100.0 \\
183.0\end{array}$ & 54.6 & 16.7 & $\begin{array}{l}0.9 \\
1.4\end{array}$ & $\begin{array}{l}100.0 \\
1675\end{array}$ & 59.7 & \\
\hline \multirow{3}{*}{ (+)-limonene } & intensity abs. & 2252 & 260 & 10000 & 888003 & 1914796 & 1402716 & 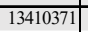 & 161307 & 9060763 & 6012873 & 2092995 & 7314 & 2453586 & 1681518 & 276305 \\
\hline & intensity \% & 22.5 & 2.6 & 100.0 & 6.6 & 14.3 & 10.5 & 100.0 & 1.8 & 100.0 & 66.4 & 23.1 & 0.3 & 100.0 & 68.5 & 11.3 \\
\hline & & 22.5 & 2.6 & & 6.6 & 14.3 & & & 2.7 & 150.7 & & & 0.4 & 145.9 & & \\
\hline \multirow[b]{2}{*}{$\alpha$-humulene } & $\begin{array}{l}\text { intensity abs. } \\
\text { jutensitio o }\end{array}$ & 440 & 80 & 10000 & $\begin{aligned} 9425117 \\
908\end{aligned}$ & 6774845 & 1669903 & 10375464 | & 1115515 & $\begin{array}{l}35079034 \\
1000\end{array}$ & 8885202 & 3453805 & 66570 & 5578126 & 1403015 & 551592 \\
\hline & $\begin{array}{l}\text { intensity \% } \\
\text { M/F\% }\end{array}$ & $\begin{array}{l}4.4 \\
4.4\end{array}$ & 0.8 & 100.0 & $\begin{array}{l}90.8 \\
908\end{array}$ & $\begin{array}{l}65.3 \\
653 \\
\end{array}$ & 16.1 & 100.0 & $\begin{array}{r}3.2 \\
126\end{array}$ & $\begin{array}{l}100.0 \\
394.8\end{array}$ & 25.3 & 9.8 & $\begin{array}{l}1.2 \\
47\end{array}$ & $\begin{array}{l}100.0 \\
3976\end{array}$ & 25.2 & 9.9 \\
\hline \multirow{3}{*}{ B-caryophyllene } & nsity abs. & 651 & $\frac{0.6}{110}$ & 10000 & 14428315 & 6281233 & 2221230 & 9929724 & 1414356 & 25684169 & 7125460 & 3271934 & $\frac{20661}{7061}$ & 4827389 & 1382543 & 613694 \\
\hline & intensity \% & 6.5 & 1.1 & 100.0 & 100.0 & 43.5 & 15.4 & 68.8 & 5.5 & 100.0 & 27.7 & 12.7 & 1.5 & 100.0 & 28.6 & 12.7 \\
\hline & M/F\% & 6.5 & 1.1 & & 145.3 & 63.3 & & & 19.8 & 360.5 & & & 5.1 & 349.2 & & \\
\hline \multirow{3}{*}{ 2-undecanone } & $\begin{array}{l}\text { intensity abs. } \\
\text { intensito o. }\end{array}$ & 691 & 100 & 10000 & $\begin{aligned} 916521 \\
366\end{aligned}$ & 2504221 & 755764 & 2075908 & 98254 & 14805182 & 4357286 & 1181882 & n.d. & 2899247 & 761959 & 127997 \\
\hline & $\begin{array}{l}\text { intensity \% } \\
\text { MWF\% }\end{array}$ & $\begin{array}{l}6.9 \\
6.9\end{array}$ & $\begin{array}{l}1.0 \\
1.0\end{array}$ & 100.0 & $\begin{array}{l}36.6 \\
44.2\end{array}$ & $\begin{array}{l}100.0 \\
120.6\end{array}$ & 30.2 & 82.9 & $\begin{array}{l}0.7 \\
2.3\end{array}$ & $\begin{array}{l}103.0 \\
393.8\end{array}$ & 29.4 & 8.0 & $\begin{array}{l}0.0 \\
0.0\end{array}$ & $\begin{array}{l}100.0 \\
380.5\end{array}$ & 26.3 & \\
\hline & insity abs. & $\frac{0.9}{40}$ & & 10000 & $\frac{44.2}{333802}$ & $\begin{array}{l}120.0 \\
112978\end{array}$ & 2578134 & 8674656 & 172286 & & 10801805 & 1445812 & & & 2780872 & 352314 \\
\hline linalool & nsity \% & 0.4 & n.d. & 100.0 & 3.8 & 1.3 & 29.7 & 100.0 & $\begin{array}{l}1.6 \\
1.6\end{array}$ & n.d. & 100.0 & 13.4 & n.d. & n.d. & 100.0 & 12.7 \\
\hline \multirow[b]{2}{*}{ (+)-a-terpineol } & intensity abs. & 10 & & 10000 & 25120 & & 2492060 & 9405870 & 62988 & & 9199332 & 1524535 & & & 2442154 & 167885 \\
\hline & $\begin{array}{l}\text { intensity \% } \\
\text { MaF\% }\end{array}$ & $\begin{array}{l}0.1 \\
0.1\end{array}$ & n.d. & 100.0 & $\begin{array}{l}0.3 \\
0.3\end{array}$ & n.d. & 26.5 & 100.0 & $\begin{array}{l}0.7 \\
0.7\end{array}$ & n.d. & 100.0 & 16.6 & n.d. & n.d. & 100.0 & \\
\hline \multirow[b]{2}{*}{ cis-linalooloxide } & & & & & 38923 & 67043 & 1448637 & 2067839 | & & 81150 & 9762344 & 1544616 & & & 1401297 & \\
\hline & $\begin{array}{l}\text { intensity \% } \\
\mathbf{M} F \mathbf{F} \%\end{array}$ & n.d. & n.d. & 100.0 & $\begin{array}{l}1.9 \\
1.9\end{array}$ & $\begin{array}{l}3.2 \\
3.2\end{array}$ & 70.1 & 100.0 & n.d. & $\begin{array}{l}0.8 \\
0.8\end{array}$ & 100.0 & 15.8 & n.d. & n.d. & 100.0 & 17.4 \\
\hline \multirow{3}{*}{ (-)-caryophyllenoxide } & & 651 & 120 & 10000 & 4621720 & 2560510 & 5046993 & 3927873 & 392182 & 7961229 & 29919435 & 1971265 & 7806 & 1003932 & 4709957 & 337047 \\
\hline & & 6.5 & 1.2 & 100.0 & $\begin{array}{l}91.6 \\
916\end{array}$ & $\begin{array}{l}50.7 \\
507\end{array}$ & 100.0 & 77.8 & 1.3 & 26.6 & 100.0 & 6.6 & 0.2 & 21.3 & 100.0 & 7.2 \\
\hline & $\frac{\text { MF }}{\text { inten }}$ & 6.5 & 1.2 & 10000 & 91.6 & 50.7 & 277963 & & & & 1616996 & & 0.2 & 21.3 & 424965 & \\
\hline 2-methylbutyl isobutyrate & $\begin{array}{l}\text { intensity \% } \\
\text { MFF\% }\end{array}$ & n.d. & n.d. & 100.0 & n.d. & n.d. & 100.0 & n.d. & n.d. & n.d. & 100.0 & n.d. & n.d. & n.d. & 100.0 & n.d. \\
\hline 3-methylbutyl is obutyrate & $\begin{array}{l}\text { intensity abs. } \\
\text { intensity \% } \\
\text { MWF\% }\end{array}$ & n.d. & n.d. & $\begin{array}{l}10000 \\
100.0\end{array}$ & n.d. & n.d. & $\begin{array}{r}184307 \\
40.7\end{array}$ & $\begin{array}{r}452862 \\
100.0\end{array}$ & n.d. & n.d. & $\begin{array}{r}801260 \\
100.0\end{array}$ & $\begin{array}{r}61082 \\
7.6\end{array}$ & n.d. & n.d. & $\begin{array}{r}209123 \\
100.0\end{array}$ & n.d. \\
\hline is sobutyl isobutyrate & $\begin{array}{l}\text { intensity abs. } \\
\text { intensity \% } \\
\text { MFF\% }\end{array}$ & n.d. & n.d. & $\begin{array}{l}10000 \\
100.0\end{array}$ & n.d. & n.d. & $\begin{array}{r}491807 \\
100.0\end{array}$ & n.d. & n.d. & n.d. & $\begin{array}{r}1546459 \\
100.0\end{array}$ & n.d. & n.d. & n.d. & $\begin{array}{r}48826 \\
100.0\end{array}$ & n.d. \\
\hline butyl isobutyrate & $\begin{array}{l}\text { intensity abs. } \\
\text { intensity \% } \\
\text { MF\%\% }\end{array}$ & n.d. & n.d. & $\begin{array}{l}10000 \\
100.0\end{array}$ & n.d. & n.d. & $\begin{array}{r}1018440 \\
100.0\end{array}$ & n.d. & n.d. & n.d. & $\begin{array}{r}2725503 \\
100.0\end{array}$ & n.d. & n.d. & n.d. & $\begin{array}{r}824950 \\
100.0\end{array}$ & n.d. \\
\hline 2-methylbutyl 2-methylbutyrate & $\begin{array}{l}\text { sity abs. } \\
\text { sity \% } \\
\% \\
\%\end{array}$ & $\begin{array}{r}60 \\
0.6 \\
0.6 \\
\end{array}$ & n.d. & $\begin{array}{r}10000 \\
100.0\end{array}$ & n.d. & n.d. & $\begin{array}{r}899185 \\
100.0\end{array}$ & n.d. & n.d. & n.d. & $\begin{array}{r}3821190 \\
100.0\end{array}$ & n.d. & n.d. & n.d. & $\begin{array}{r}867655 \\
100.0\end{array}$ & n.d. \\
\hline 2-methylbutyl 3-methylbutyrate & $\begin{array}{l}\text { intensity abs. } \\
\text { intensity \% } \\
\text { MFF\% }\end{array}$ & $\begin{array}{l}20 \\
0.2 \\
0.2 \\
\end{array}$ & n.d. & $\begin{array}{c}10000 \\
100.0\end{array}$ & n.d. & n.d. & $\begin{array}{r}827250 \\
100.0\end{array}$ & n.d. & n.d. & n.d. & $\begin{array}{r}3656261 \\
100.0\end{array}$ & n.d. & n.d. & n.d. & $\begin{array}{r}783148 \\
100.0 \\
\end{array}$ & n.d. \\
\hline propyl 2-methylbutyrate & $\begin{array}{l}\text { intensity abs. } \\
\text { intensity \% } \\
\text { M/F\%\% }\end{array}$ & n.d. & n.d. & $\begin{array}{l}10000 \\
100.0\end{array}$ & n.d. & $\begin{array}{r}6656 \\
0.2 \\
0.2\end{array}$ & $\begin{array}{r}2861967 \\
100.0\end{array}$ & n.d. & n.d. & $\begin{array}{r}56370 \\
0.8 \\
0.8\end{array}$ & $\begin{array}{r}7273267 \\
100.0\end{array}$ & n.d. & n.d. & $\begin{array}{r}36944 \\
2.1 \\
2.1\end{array}$ & $\begin{array}{r}1727999 \\
100.0\end{array}$ & n.d. \\
\hline & & 90 & 10 & $1000 \mathrm{C}$ & 28139 & 7463009 & 216536 & 271237 & & 22919683 & 708737 & 69909 & & 5517095 & 162290 & \\
\hline methyl 2-methylbutyrate & $\frac{\text { int }}{\mathrm{M} /}$ & $\begin{array}{l}0.9 \\
0.9\end{array}$ & $\begin{array}{l}0.1 \\
0.1 \\
0.1\end{array}$ & 100.0 & $\begin{array}{r}0.4 \\
10.4 \\
\end{array}$ & $\begin{array}{r}100.0 \\
2751.5 \\
\end{array}$ & 2.9 & 3.6 & n.d. & $\begin{array}{r}100.0 \\
3233.9 \\
\end{array}$ & 3.1 & 0.3 & n.d. & $\begin{array}{r}100.0 \\
3399.5 \\
\end{array}$ & 2.9 & n.d. \\
\hline & & 140 & 20 & 10000 & 1023525 & 1754949 & 9516793 & & 121411 & 9343844 & 21842259 & & & 1667046 & 3673415 & \\
\hline methyl geranate & & 1.4 & 0.2 & 100.0 & 10.8 & 18.4 & 100.0 & n.d. & 0.6 & 42.8 & 100.0 & n.d. & n.d. & 45.4 & 100.0 & n.d. \\
\hline & & $\frac{1.4}{10}$ & 0.2 & & & & & & 0.6 & & & & & 45.4 & & \\
\hline geranyl acetate & $\begin{array}{l}\text { nsity abs. } \\
\text { nisity \% } \\
\text { \%o }\end{array}$ & $\begin{array}{l}10 \\
0.1 \\
0.1\end{array}$ & n.d. & $\begin{array}{r}10000 \\
100.0\end{array}$ & $\begin{array}{r}37788 \\
0.5 \\
0.5\end{array}$ & $\begin{array}{r}15778 \\
0.2 \\
0.2\end{array}$ & $\begin{array}{r}2988514 \\
40.7\end{array}$ & $\begin{array}{r}7341491 \\
100.0\end{array}$ & n.d. & $\begin{array}{r}51175 \\
0.3 \\
0.3\end{array}$ & $\begin{array}{r}14730445 \\
100.0\end{array}$ & $\begin{array}{r}1237604 \\
8.4\end{array}$ & n.d. & n.d. & $\begin{array}{r}3089285 \\
100.0\end{array}$ & $\begin{aligned} 243278 \\
7.9\end{aligned}$ \\
\hline & & & & 10000 & & & 2192033 & 6717874 & & & 11863052 & 1278993 & & & 2010975 & \\
\hline geranyl propionate & $\begin{array}{l}\text { intensity \% } \\
\text { MFF\% }\end{array}$ & n.d. & n.d. & 100.0 & $\begin{array}{l}0.7 \\
0.7 \\
\end{array}$ & $\begin{array}{l}0.3 \\
0.3 \\
\end{array}$ & 32.6 & 100.0 & n.d. & $\begin{array}{r}0.6 \\
0.6 \\
\end{array}$ & 100.0 & 10.8 & n.d. & n.d. & 100.0 & n.d. \\
\hline geranyl is obutyrate & $\begin{array}{l}\text { intensity abs. } \\
\text { intensity \% } \\
\text { MFF\% }\end{array}$ & $\begin{array}{r}681 \\
6.8 \\
6.8 \\
\end{array}$ & $\begin{array}{l}110 \\
1.1 \\
1.1 \\
\end{array}$ & $\begin{array}{l}10000 \\
100.0\end{array}$ & $\begin{array}{r}68486 \\
1.6 \\
1.6 \\
\end{array}$ & $\begin{array}{r}27432 \\
0.6 \\
0.6 \\
\end{array}$ & $\begin{array}{r}1414773 \\
32.3\end{array}$ & $\begin{array}{r}4376524 \\
100.0\end{array}$ & n.d. & $\begin{array}{r}71644 \\
1.1 \\
1.1 \\
\end{array}$ & $\begin{array}{r}6800898 \\
100.0\end{array}$ & $\begin{array}{r}737923 \\
10.9 \\
\end{array}$ & n.d. & n.d. & $\begin{array}{r}882922 \\
100.0 \\
\end{array}$ & n.d. \\
\hline
\end{tabular}

n.d.: ion not detected

F80 and F200: fragment ions at cone gas flows of 80 and $200 \mathrm{~L} / \mathrm{h}$, respectively. 


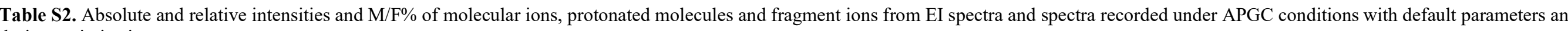
during optimization.

\begin{tabular}{|c|c|c|c|c|c|c|c|c|c|c|c|c|c|c|c|c|c|}
\hline \multirow{3}{*}{ analyte } & & \multicolumn{16}{|c|}{ 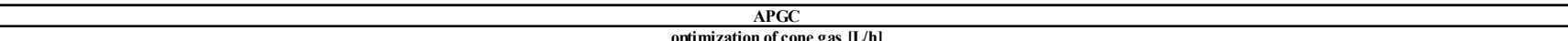 } \\
\hline & & \multicolumn{4}{|c|}{100} & \multicolumn{4}{|c|}{ optimization of } & \multicolumn{4}{|l|}{ ne gas $[\mathrm{L} / \mathrm{h}]$} & \multicolumn{4}{|c|}{160} \\
\hline & & {$[\mathbf{M i}]^{+}$} & {$\left[\mathbf{M}+\mathbf{H}^{+}\right.$} & F80 & F200 & {$\left[M^{i}\right]^{+}$} & {$\left[\mathbf{M}+\mathbf{H}^{+}\right.$} & $\mathbf{F 8 0}$ & F200 & {$\left[\mathrm{Mi}^{+}\right]^{+}$} & {$\left[\mathbf{M}+\mathrm{H}^{+}\right.$} & & F200 & $\mathrm{Fi}^{+}$ & {$\left[\mathbf{M}+\mathrm{H}^{+}\right.$} & & F200 \\
\hline \multirow{3}{*}{ myrcene } & intensity abs. & 209826 & & 6516130 & & 270812 & & 5205766 & \begin{tabular}{|c|c|}
1737538 \\
\end{tabular} & 569743 & 8533735 & 4752664 & & $\frac{1145517}{114}$ & & 389656 & 10358812 \\
\hline & intensity \% & 1.8 & 100.0 & 54.8 & 11.1 & 3.0 & 100.0 & 56.9 & 19.0 & 6.7 & 100.0 & 55.7 & 41.0 & 11.1 & 3.5 & 3.8 & 100.0 \\
\hline & & 3.2 & 182.6 & & & 5.2 & 175.9 & & & 12.0 & 179.6 & & & 11.1 & 3.5 & & \\
\hline \multirow[b]{2}{*}{$(-)-\beta$-pinene } & intensity abs. & 254077 & 16030660 & 8641814 & 1450771 & 365128 & 12747149 & 6922285 & 2158877 & 759840 & 11626967 & 6165824 & 4519679 & 2132722 & 518729 & 399950 & 15450428 \\
\hline & $\begin{array}{l}\text { intensity \% } \\
\text { M/F\% }\end{array}$ & $\begin{array}{l}1.6 \\
29\end{array}$ & $\begin{array}{l}100.0 \\
185\end{array}$ & 53.9 & & $\begin{array}{l}2.9 \\
5.3\end{array}$ & $\begin{array}{l}100.0 \\
184.1\end{array}$ & 54.3 & 16.9 & $\begin{array}{r}6.5 \\
12.3\end{array}$ & $\begin{array}{l}100.0 \\
188.6\end{array}$ & 53.0 & 38.9 & $\begin{array}{l}13.8 \\
13.8\end{array}$ & $\begin{array}{l}3.4 \\
3.4\end{array}$ & 2.6 & 100.0 \\
\hline \multirow{3}{*}{ (t)-limonene } & intensity abs. & 145722 & 12989872 & 8600214 & 1620850 & 207626 & 10518049 & 6896904 & 2410078 & $\frac{12.5}{433303}$ & 93121711 & 6137845 & 5207205 & $\frac{1596}{86946}$ & 298102 & 484900 & 15389845 \\
\hline & intensity \% & 1.1 & 100.0 & 66.2 & 12.5 & 2.0 & 100.0 & 65.6 & 22.9 & 4.7 & 100.0 & 65.9 & 55.9 & 5.6 & 1.9 & 3.2 & 100.0 \\
\hline & M/F\% $\%$ & 1.7 & 151.0 & & & 3.0 & 152.5 & & & 7.1 & 151.7 & & & 5.6 & 1.9 & & \\
\hline \multirow{3}{*}{ a-humulene } & intensity abs. & 1056828 & 43893366 & 11481255 & 3914258 & 1562815 & 41117800 & 10515981 & 4154012 & 3763097 & 37811766 & 9571192 & 5693600 & 9655253 & 2292324 & 775983 & 11986475 \\
\hline & intensity \% & 2.4 & 100.0 & 26.2 & 8.9 & 3.8 & 100.0 & 25.6 & 10.1 & 10.0 & 100.0 & 25.3 & 15.1 & 80.6 & 19.1 & 6.5 & 100.0 \\
\hline & M/F\% & & 382.3 & & & 14.9 & 391.0 & & & 39.3 & 395.1 & & & 80.6 & 19.1 & & \\
\hline \multirow[b]{2}{*}{ B-caryophyllene } & $\begin{array}{l}\text { intensity abs. } \\
\text { intensitio } \% \text {. }\end{array}$ & $\begin{array}{r}1396607 \\
37\end{array}$ & & 10645108 & 4202490 & 2250398 & & $\begin{array}{r}9715665 \\
275\end{array}$ & 4453047 & & 32232506 & 9019045 & & & & 1499508 & 12121046 \\
\hline & $\begin{array}{l}\text { intensity \% } \\
\text { MF\% }\end{array}$ & $\begin{array}{r}3.7 \\
13.1\end{array}$ & $\begin{array}{l}100.0 \\
357.6\end{array}$ & 28.0 & 11.0 & $\begin{array}{r}6.4 \\
232\end{array}$ & $\begin{array}{l}100.0 \\
3630\end{array}$ & 27.5 & 12.6 & $\begin{array}{l}16.8 \\
599\end{array}$ & $\begin{array}{l}100.0 \\
3574\end{array}$ & 28.0 & 18.7 & 100.0 & 21.0 & 9.9 & 79.9 \\
\hline \multirow{3}{*}{ 2-undecanone } & intensity abs. & 103760 & 20885889 & 5666460 & 1272184 & $\frac{25.2}{169228}$ & $\begin{array}{l}303.0 \\
19060769\end{array}$ & 5412206 & 1313181 & 399920 & $\begin{aligned} 35 / .4 \\
17624598\end{aligned}$ & 5047592 & 1650070 & $\frac{125.1}{1065053}$ & 602.2 & 222382 & 2601661 \\
\hline & intensity \% & 0.5 & 100.0 & 27.1 & 6.1 & 0.9 & 100.0 & 28.4 & 6.9 & 2.3 & 100.0 & 28.6 & 9.4 & 38.2 & 24.4 & 8.0 & 93.2 \\
\hline & M/F\% & 1.8 & 368.6 & & & 3.1 & 352.2 & & & 7.9 & 349.2 & & & 40.9 & 26.1 & & \\
\hline \multirow[b]{2}{*}{ linalool } & intensity abs. & & & 18195983 & $\begin{array}{ll}1664518 \\
\end{array}$ & 264533 & & 14832071 & 2016581 & 370986 & 238690 & 12690489 & 3286808 & 300475 & 50586 & 600399 & 10225334 \\
\hline & $\begin{array}{l}\text { intensity \% } \\
\text { M/FF\% }\end{array}$ & n.d. & n.d. & 100.0 & 9.1 & $\begin{array}{l}1.8 \\
1.8\end{array}$ & n.d. & 100.0 & 13.6 & $\begin{array}{l}2.9 \\
2.9\end{array}$ & $\begin{array}{l}1.9 \\
1.9\end{array}$ & 100.0 & 25.9 & $\begin{array}{l}2.9 \\
2.9\end{array}$ & $\begin{array}{l}0.5 \\
0.5\end{array}$ & 5.9 & 100.0 \\
\hline \multirow[b]{2}{*}{ (+)-a-terpineol } & intensity abs. & 18413 & & 14792770 & 1347342 & 59965 & & 11642953 & 1779916 & 147879 & 9629 & 10426224 & 3378932 & 51459 & & 839898 & 10907909 \\
\hline & $\begin{array}{l}\text { intensity \% } \\
\text { y FFo }\end{array}$ & 0.1 & n.d. & 100.0 & & $\begin{array}{l}0.5 \\
0.5\end{array}$ & n.d. & 100.0 & 15.3 & $\begin{array}{r}1.4 \\
1.4\end{array}$ & 0.1 & 100.0 & 32.4 & $\begin{array}{l}0.5 \\
0.5\end{array}$ & n.d. & 7.7 & 100.0 \\
\hline \multirow{3}{*}{ cis-linalooloxide } & intensity abs. & & 71380 & 11568686 & 1345190 & & 73801 & 11570438 & 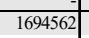 & 23283 & $\frac{0.1}{123471}$ & 10130987 & 1886118 & 44800 & 23134 & 340191 & 3122902 \\
\hline & $\begin{array}{l}\text { intensity \% } \\
\text { W/F\% }\end{array}$ & n.d. & $\begin{array}{l}0.6 \\
0.6\end{array}$ & 100.0 & 11.6 & n.d. & $\begin{array}{l}0.6 \\
0.6\end{array}$ & 100.0 & 14.6 & $\begin{array}{l}0.2 \\
0.2\end{array}$ & $\begin{array}{l}1.2 \\
1.2\end{array}$ & 100.0 & 18.6 & $\begin{array}{l}1.4 \\
1.4\end{array}$ & $\begin{array}{l}0.7 \\
0.7\end{array}$ & 10.9 & 100.0 \\
\hline & intensity abs. & 343978 & 11199993 & 40528336 & 2327720 & 604486 & 10781888 & 41438016 & 2506354 & 1504118 & 10794406 & 38153248 & 2884097 & $\begin{array}{r}1.4 \\
5543738\end{array}$ & 1412332 & 1845027 & 4764193 \\
\hline \multirow{2}{*}{ (-)-caryophyllenoxide } & intensity \% & 0.8 & 27.6 & 100.0 & 5.7 & 1.5 & 26.0 & 100.0 & 6.0 & 3.9 & 28.3 & 100.0 & 7.6 & 100.0 & 25.5 & 33.3 & 85.9 \\
\hline & M/F\% & 0.8 & 27.6 & & & 1.5 & 26.0 & & & 3.9 & 28.3 & & & 116.4 & 29.6 & & \\
\hline 2-methylbutyl isobutyrate & $\begin{array}{l}\text { intensity abs. } \\
\text { intensity \% } \\
\text { y/FF\% }\end{array}$ & n.d. & n.d. & $\begin{array}{r}2230259 \\
100.0\end{array}$ & n.d. & n.d. & n.d. & $\begin{array}{r}2006069 \\
100.0\end{array}$ & n.d. & n.d. & n.d. & $\begin{array}{r}2115287 \\
100.0\end{array}$ & n.d. & $\begin{array}{r}10406 \\
6.0 \\
60\end{array}$ & n.d. & $\begin{array}{r}174398 \\
100.0\end{array}$ & n.d. \\
\hline 3-methylbutyl isobutyrate & $\begin{array}{l}\text { intensity abs. } \\
\text { intensity \% } \\
\mathbf{M} / \mathbf{F} \%\end{array}$ & n.d. & n.d. & $\begin{array}{r}1135626 \\
100.0\end{array}$ & $\begin{array}{r}57486 \\
5.1\end{array}$ & n.d. & n.d. & $\begin{array}{r}1021823 \\
100.0\end{array}$ & $\begin{array}{r}87764 \\
8.6\end{array}$ & n.d. & n.d. & $\begin{array}{r}671503 \\
100.0\end{array}$ & $\begin{array}{r}555446 \\
82.7\end{array}$ & n.d. & n.d. & $\begin{array}{r}151526 \\
29.0 \\
\end{array}$ & $\begin{array}{r}523243 \\
100.0\end{array}$ \\
\hline is obutyl isobutyrate & $\begin{array}{l}\text { intensity abs. } \\
\text { intensity \% } \\
\text { M/F\% } \\
\end{array}$ & n.d. & n.d. & $\begin{array}{r}1974445 \\
100.0\end{array}$ & n.d. & n.d. & n.d. & $\begin{array}{r}1693000 \\
100.0\end{array}$ & n.d. & n.d. & n.d. & $\begin{array}{r}1757289 \\
100.0\end{array}$ & n.d. & n.d. & n.d. & $\begin{array}{r}296348 \\
100.0\end{array}$ & n.d. \\
\hline butyl isobutyrate & $\begin{array}{l}\text { intensity abs. } \\
\text { intensity \% } \\
\text { M/F\% }\end{array}$ & n.d. & n.d. & $\begin{array}{r}3911600 \\
100.0\end{array}$ & n.d. & n.d. & n.d. & $\begin{array}{r}3456790 \\
100.0\end{array}$ & n.d. & n.d. & n.d. & $\begin{array}{r}3375038 \\
100.0\end{array}$ & n.d. & n.d. & n.d. & $\begin{array}{r}682925 \\
100.0\end{array}$ & n.d. \\
\hline 2-methylbutyl 2-methylbutyrate & $\begin{array}{l}\text { intensity abs. } \\
\text { intensity \% } \\
\text { M/F\% } \\
\end{array}$ & n.d. & n.d. & $\begin{array}{r}4848765 \\
100.0\end{array}$ & n.d. & n.d. & n.d. & $\begin{array}{r}5453938 \\
100.0\end{array}$ & n.d. & n.d. & n.d. & $\begin{array}{r}4680875 \\
100.0\end{array}$ & n.d. & n.d. & n.d. & $\begin{array}{r}439564 \\
100.0\end{array}$ & n.d. \\
\hline 2-methylbutyl 3-methylbutyrate & $\begin{array}{l}\text { intensity abs. } \\
\text { intensity \% } \\
\text { M/F\% }\end{array}$ & n.d. & n.d. & $\begin{array}{r}5125234 \\
100.0\end{array}$ & n.d. & n.d. & n.d. & $\begin{array}{r}4685171 \\
100.0\end{array}$ & n.d. & n.d. & n.d. & $\begin{array}{r}4762445 \\
100.0\end{array}$ & n.d. & n.d. & n.d. & $\begin{array}{r}391721 \\
100.0\end{array}$ & n.d. \\
\hline propyl 2-methylbutyrate & $\begin{array}{l}\text { intensity abs. } \\
\text { intensity \% } \\
\text { M/F\% }\end{array}$ & n.d. & $\begin{array}{r}60223 \\
0.7 \\
0.7\end{array}$ & $\begin{array}{r}8907946 \\
100.0\end{array}$ & n.d. & n.d. & $\begin{array}{r}56221 \\
0.7 \\
0.7\end{array}$ & $\begin{array}{r}7764587 \\
100.0\end{array}$ & n.d. & n.d. & $\begin{array}{r}40142 \\
0.5 \\
0.5\end{array}$ & $\begin{array}{r}7983355 \\
100.0\end{array}$ & n.d. & n.d. & n.d. & $\begin{array}{r}2054275 \\
100.0\end{array}$ & n.d. \\
\hline & intensity abs. & & 28402441 & 914941 & 41793 & & 26340502 & 830306 & 66851 & 16892 & 28593532 & 916675 & 125070 & 30087 & 1390584 & 45302 & 211744 \\
\hline methyl 2-methylbutyrate & intensity \% & n.d. & $\begin{array}{r}100.0 \\
3303\end{array}$ & 3.2 & 0.1 & n.d. & $\begin{array}{r}100.0 \\
31724\end{array}$ & 3.2 & 0.3 & 0.1 & $\begin{array}{r}100.0 \\
3103\end{array}$ & 3.2 & 0.4 & 2.2 & $\begin{array}{l}100.0 \\
6567\end{array}$ & 3.3 & 15.2 \\
\hline & & & 3104.3 & & & & 3172.4 & & & 1.8 & 3119.3 & & & 14.2 & 656.7 & & \\
\hline & intensity abs. & 118768 & 13062329 & 29940334 & & 169340 & 11547406 & 26806306 & & 419946 & 10509748 & 25016014 & & 1209198 & 484904 & 7277639 & \\
\hline methyl geranate & $\begin{array}{l}\text { intensity \% } \\
\text { M/F\% }\end{array}$ & 0.4 & $\begin{array}{l}43.6 \\
436\end{array}$ & 100.0 & n.d. & 0.6 & $\begin{array}{l}43.1 \\
43\end{array}$ & 100.0 & n.d. & 1.7 & $\begin{array}{ll}42.0 \\
420\end{array}$ & 100.0 & n.d. & $\begin{array}{l}16.6 \\
166\end{array}$ & $\begin{array}{l}6.7 \\
67\end{array}$ & 100.0 & n.d. \\
\hline & $\begin{array}{l}\text { M/F\% } \\
\text { intensity abs. }\end{array}$ & & $\begin{array}{r}4386 \\
82866\end{array}$ & 23999239 & $\mid 1239712$ & & $\frac{43.1}{74218}$ & 19663976 & 1506585 & $\frac{1.7}{9392}$ & $\frac{42.0}{74509}$ & 16981571 & & $\frac{16.6}{41608}$ & & 1119439 & 8627226 \\
\hline geranyl acetate & intensity \% & n.d. & $\begin{array}{r}02000 \\
0.3\end{array}$ & 100.0 & 5.2 & n.d. & $\begin{array}{r}r 410 \\
0.4\end{array}$ & 100.0 & & 0.1 & 0.4 & 100.0 & 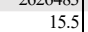 & $\begin{array}{r}41000 \\
0.5\end{array}$ & $n d$ & 年 & 年 \\
\hline & & & 0.3 & & & & 0.4 & & & 0.1 & 0.4 & & & 0.5 & m.co: & & \\
\hline & intensity abs. & & 98260 & 19604064 & 1058035 & & 94234 & 16290068 & 1525653 & 22990 & 105832 & 14435984 & 2505513 & 62781 & & 840136 & 8065785 \\
\hline geranyl propionate & $\begin{array}{l}\text { intensity \% } \\
y \text { yFo }\end{array}$ & n.d. & $\begin{array}{l}0.5 \\
0.5\end{array}$ & 100.0 & & n.d. & $\begin{array}{l}0.6 \\
06\end{array}$ & 100.0 & 9.4 & 0.2 & 0.7 & 100.0 & 17.4 & 0.8 & n.d. & 10.4 & 100.0 \\
\hline & $\frac{\text { M/F\% } \%}{\text { intensity abs. }}$ & & $\begin{array}{l}0.5 \\
117215\end{array}$ & 11575222 & 761262 & $\overline{11328}$ & $\frac{0.6}{121294}$ & 9342951 & 1005628 & $\frac{0.2}{30768}$ & $\begin{array}{l}0.7 \\
134267\end{array}$ & 8307222 & $\mid \begin{array}{ll}1664666 \\
\end{array}$ & $\frac{0.8}{84627}$ & 9991 & 611929 & 5030757 \\
\hline geranyl is obutyrate & intensity \% & n.d. & 1.0 & 100.0 & 6.6 & 0.1 & 1.3 & 100.0 & 10.8 & 0.4 & 1.6 & 100.0 & 20.0 & 1.7 & 0.2 & 12.2 & 100.0 \\
\hline & M/F\% & & 1.0 & & & 0.1 & 1.3 & & & 0.4 & 1.6 & & & 1.7 & 0.2 & & \\
\hline
\end{tabular}

n.d.: ion not detected

F80 and F200: fragment ions at cone gas flows of 80 and $200 \mathrm{~L} / \mathrm{h}$, respectively. 


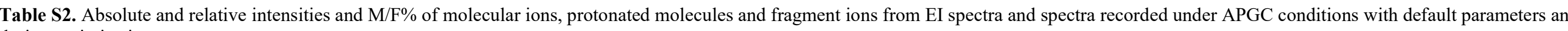
during optimization.

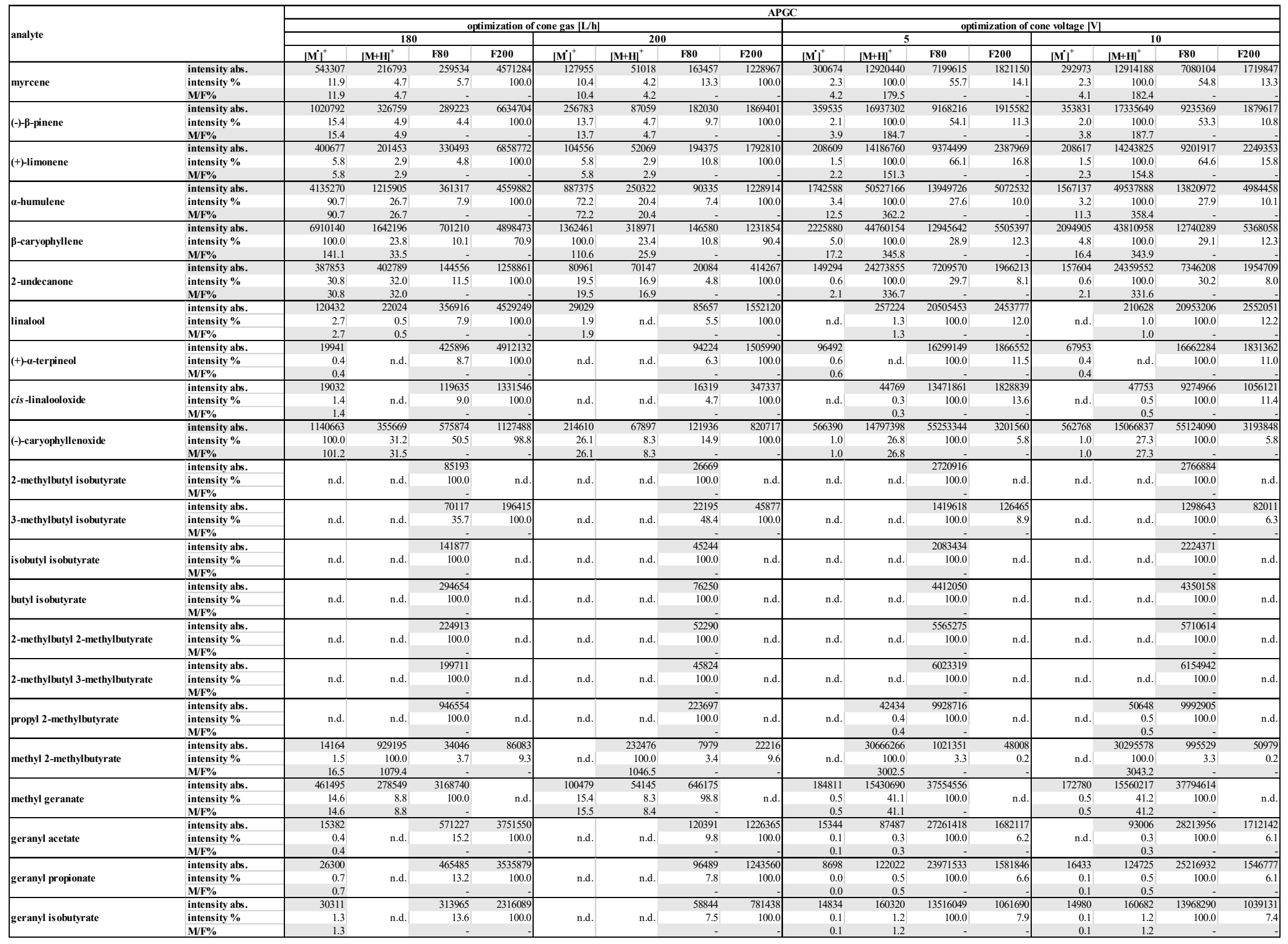

n.d.: ion not detected

F80 and F200: fragment ions at cone gas flows of 80 and $200 \mathrm{~L} / \mathrm{h}$, respectively. 


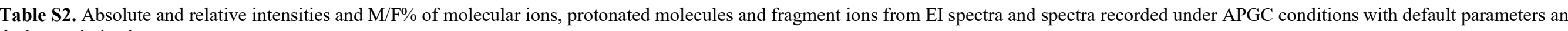
during optimization.

\begin{tabular}{|c|c|c|c|c|c|c|c|c|c|c|c|c|c|c|c|c|c|}
\hline \multirow{3}{*}{ analyte } & & \multirow{2}{*}{\multicolumn{16}{|c|}{$\begin{array}{l}\text { APGC } \\
\text { ation of cone voltage [V] }\end{array}$}} \\
\hline & & \multicolumn{4}{|c|}{15} & \multicolumn{4}{|r|}{ zation of } & \multicolumn{2}{|l|}{ ie voltage $|\mathrm{V}|$} & & & \multicolumn{4}{|c|}{30} \\
\hline & & {$\left[\mathrm{Mi}^{\mathbf{+}}{ }^{+}\right.$} & $\mathbf{M + \mathbf { H } ^ { + }}$ & F80 & F200 & {$\left[\mathbf{M}^{+}\right]^{+}$} & {$[\mathbf{M}+\mathbf{H}]^{+}$} & F80 & F200 & {$\left[\mathbf{M}^{\theta^{+}}{ }^{-}\right.$} & {$\left[\mathbf{M}+\mathbf{H}^{+}\right.$} & F80 & F200 & {$\left[\mathbf{M i}^{+}\right]^{+}$} & & F80 & F200 \\
\hline \multirow{3}{*}{ myrcene } & intensity abs. & 303783 & $\frac{1 \times 1+11}{13390364}$ & 7230769 & 1724931 & $\frac{111}{255048}$ & $\frac{11+1+1}{12073012}$ & 6568580 & 1472754 & 256046 & $\frac{|N+1| 10912}{12010912}$ & 6467349 & $\frac{\pi 210038}{147638}$ & $\frac{1 \mathrm{M} 1}{239568}$ & 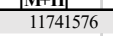 & 6276317 & $\frac{F 200}{1429122}$ \\
\hline & intensity \% & 2.3 & 100.0 & 54.0 & 12.9 & 2.1 & 100.0 & 54.4 & 12.2 & 2.1 & 100.0 & 53.8 & 12.3 & 2.0 & 100.0 & 53.5 & 12.2 \\
\hline & M/F\% & 4.2 & 185.2 & & & 3.9 & 183.8 & & & 4.0 & 185.7 & & & 3.8 & 187.1 & & \\
\hline \multirow{2}{*}{ (-)-p-pinene } & $\begin{array}{l}\text { intensity abs. } \\
\text { intensitio }\end{array}$ & 330149 & $\begin{array}{r}17213122 \\
1000\end{array}$ & $\begin{array}{l}9048864 \\
556\end{array}$ & 1828359 & 309444 & $\begin{array}{r}15931677 \\
1000\end{array}$ & 8459592 & 1569362 & 306586 & 16151725 & 8550811 & 1636411 & 302563 & 15926632 & 8281987 & 1596083 \\
\hline & $\begin{array}{l}\text { intensity \% } \\
\text { M/F\% }\end{array}$ & $\begin{array}{l}1.99 \\
3.6\end{array}$ & $\begin{array}{l}109.0 \\
190.2\end{array}$ & 52.6 & 10.6 & $\begin{array}{l}1.9 \\
3.7\end{array}$ & $\begin{array}{l}100.0 \\
188.3\end{array}$ & 53.1 & 9.9 & $\begin{array}{l}1.9 \\
3.6\end{array}$ & $\begin{array}{l}100.0 \\
188.9\end{array}$ & 52.9 & 10.1 & $\begin{array}{l}1.9 \\
3.7\end{array}$ & $\begin{array}{l}100.0 \\
1923\end{array}$ & 52.0 & 10.0 \\
\hline \multirow{3}{*}{ (+)-limonene } & intensity abs. & 203495 & 14297463 & 9155905 & 2200538 & 164650 & 13128305 & 8520838 & 1846963 & 174309 & 13167887 & 8486456 & 1848619 & 162350 & 12642695 & 8042938 & 1803097 \\
\hline & intensity \% & 1.4 & & 64.0 & 15.4 & 1.3 & 100.0 & 64.9 & 14.1 & 1.3 & 100.0 & 64.4 & 14.0 & 1.3 & 100.0 & 63.6 & 14.3 \\
\hline & MF\% & 2.2 & & & & 1.9 & 154.1 & & & 2.1 & 155.2 & & & 2.0 & 157.2 & & \\
\hline \multirow{2}{*}{ a-humulene } & $\begin{array}{l}\text { intensity abs. } \\
\text { intensitio o }\end{array}$ & 1572125 & 51015522 & 13726253 & 4914409 & 1335126 & 45086540 & 11960609 & 4251586 & 1368808 & 47370146 & 12495099 & 4437889 & 1317738 & 46816334 & 12374794 & 4359771 \\
\hline & $\begin{array}{l}\text { intensity \% } \\
\text { M/F\% }\end{array}$ & $\begin{array}{r}3.1 \\
11.5\end{array}$ & $\begin{array}{l}100.0 \\
377.7\end{array}$ & & 9.6 & $\begin{array}{r}3.0 \\
112\end{array}$ & $\begin{array}{l}100.0 \\
3770\end{array}$ & 26.5 & 9.4 & $\begin{array}{r}2.9 \\
110\end{array}$ & $\begin{array}{l}100.0 \\
379 .\end{array}$ & 26.4 & 9.4 & 2.8 & $\begin{array}{l}100.0 \\
3783\end{array}$ & & 9.3 \\
\hline \multirow[b]{2}{*}{ B-caryophyllene } & intensity abs. & 2054743 & 44732036 & 12699444 & 5216139 & 1743264 & 39949290 & 11203448 & 4535510 & $\frac{11.0}{1860617}$ & $\frac{519.1}{4200734}$ & 11712581 & 4766524 & 1768617 & $\frac{31472720}{41420}$ & 11543109 & 4635078 \\
\hline & intensity \% & $\begin{array}{r}4.6 \\
\end{array}$ & $\begin{array}{l}100.0 \\
352\end{array}$ & 28.4 & 11.7 & $\begin{array}{r}4.4 \\
156\end{array}$ & 100.0 & 28.0 & 11.4 & 4.4 & 100.0 & 27.9 & 11.3 & 4.3 & 100.0 & 27.8 & 11.2 \\
\hline \multirow{3}{*}{ 2-undecanone } & intensity abs. & $\frac{10.2}{149137}$ & $\frac{32.2}{23681697}$ & 7344014 & 2095851 & $\frac{15.0}{129780}$ & $\frac{300.0}{21444237}$ & $\begin{array}{l}6458586 \\
\end{array}$ & 1956314 & $\frac{15.99}{14139}$ & $\frac{388.1}{22283405}$ & 6728495 & $\mid$ & $\frac{15.3}{130038}$ & $\frac{359.3}{2185319}$ & 6702906 & 1401008 \\
\hline & intensity \% & 0.6 & 100.0 & 31.0 & 8.9 & 0.6 & 100.0 & 30.1 & 9.1 & 0.6 & 100.0 & 30.2 & 7.6 & 0.6 & 100.0 & 30.7 & 6.4 \\
\hline & $\frac{\text { M/F\% }}{\text { intensity abs. }}$ & & $\frac{322.5}{202235}$ & 21211669 & 2381399 & & $\frac{332.0}{185237}$ & 18801535 & 2182698 & 2.1 & $\frac{331.2}{2230202}$ & 19915500 & 2362087 & 1.9 & $\frac{326.0}{244895}$ & 19449014 & 2338681 \\
\hline linalool & $\begin{array}{l}\text { intensity \% } \\
\text { M/F\% }\end{array}$ & n.d. & $\begin{array}{l}1.0 \\
1.0 \\
\end{array}$ & 100.0 & 11.2 & n.d. & $\begin{array}{l}1.0 \\
1.0 \\
\end{array}$ & 100.0 & 11.6 & n.d. & $\begin{array}{l}1.1 \\
1.1 \\
\end{array}$ & 100.0 & 11.9 & n.d. & $\begin{array}{l}1.3 \\
1.3 \\
\end{array}$ & 100.0 & 12.0 \\
\hline (+)-a-terpineol & $\begin{array}{l}\text { intensity abs. } \\
\text { intensity \% } \\
\text { MFF\% }\end{array}$ & $\begin{array}{r}79201 \\
0.5 \\
0.5\end{array}$ & n.d. & $\begin{array}{r}16453688 \\
100.0\end{array}$ & $\begin{array}{r}1844543 \\
11.2\end{array}$ & $\begin{array}{r}66268 \\
0.4 \\
04\end{array}$ & n.d. & $\begin{array}{r}15008442 \\
100.0\end{array}$ & $\begin{array}{r}1591759 \\
10.6\end{array}$ & $\begin{array}{r}72584 \\
0.5 \\
0.5\end{array}$ & n.d. & $\begin{array}{r}15440390 \\
100.0\end{array}$ & $\begin{array}{r}1614886 \\
10.5\end{array}$ & $\begin{array}{r}55146 \\
0.4 \\
04\end{array}$ & n.d. & $\begin{array}{r}15441222 \\
100.0\end{array}$ & $\begin{array}{r}1612351 \\
10.4\end{array}$ \\
\hline \multirow{2}{*}{ cis-linalooloxide } & intensity abs. & & 60569 & 14112391 & 2457033 & & 45468 & 8587891 & 1004821 & & 66910 & 12896508 & 929554 & & 44821 & 13091514 & 1943637 \\
\hline & $\begin{array}{l}\text { intensity \% } \\
\text { M/F\% }\end{array}$ & n.d. & $\begin{array}{l}0.4 \\
0.4\end{array}$ & 100.0 & 17.4 & n.d. & $\begin{array}{l}0.5 \\
0.5\end{array}$ & 100.0 & 11.7 & n.d. & $\begin{array}{l}0.5 \\
0.5\end{array}$ & 100.0 & 7.2 & n.d. & $\begin{array}{l}0.3 \\
0.3\end{array}$ & 100.0 & 14.8 \\
\hline \multirow{3}{*}{ (-)-caryophyllenoxide } & intensity abs. & 518947 & 14834155 & 55324954 & 3222846 & 440670 & 13089284 & 48825454 & 2744953 & 493023 & 15128407 & 53268292 & 3064102 & 486384 & 15367946 & 53727368 & 3131536 \\
\hline & intensity \% & 0.9 & 26.8 & 100.0 & & 0.9 & 26.8 & 100.0 & 5.6 & 0.9 & 28.4 & 100.0 & 5.8 & 0.9 & 28.6 & 100.0 & 5.8 \\
\hline & $\begin{array}{l}\text { M/F\% } \% \\
\text { jutensity abr. }\end{array}$ & 0.9 & 26.8 & & & 0.9 & 26.8 & & & 0.9 & 28.4 & & & 0.9 & 28.6 & & \\
\hline 2-methylbutyl isobutyrate & $\begin{array}{l}\text { intensity abs. } \\
\text { intensity \% } \\
\text { M/F\% \% }\end{array}$ & n.d. & n.d. & $\begin{array}{r}2609808 \\
100.0\end{array}$ & n.d. & n.d. & n.d. & $\begin{array}{r}2479267 \\
100.0\end{array}$ & n.d. & n.d. & n.d. & $\begin{array}{r}2447051 \\
100.0\end{array}$ & n.d. & n.d. & n.d. & $\begin{array}{r}2369694 \\
100.0\end{array}$ & n.d. \\
\hline 3-methylbutyl isobutyrate & $\begin{array}{l}\text { intensity abs. } \\
\text { intensity \% } \\
\text { M/F\% } \\
\end{array}$ & n.d. & $\begin{array}{r}37017 \\
2.7 \\
2.7 \\
\end{array}$ & $\begin{array}{r}1353979 \\
100.0\end{array}$ & $\begin{array}{r}131848 \\
9.7\end{array}$ & n.d. & n.d. & $\begin{array}{r}1119784 \\
100.0\end{array}$ & $\begin{array}{r}96275 \\
8.6\end{array}$ & n.d. & n.d. & $\begin{array}{r}1174381 \\
100.0\end{array}$ & $\begin{array}{r}77181 \\
6.6 \\
\end{array}$ & n.d. & n.d. & $\begin{array}{r}1163948 \\
100.0\end{array}$ & $\begin{array}{r}74368 \\
6.4 \\
\end{array}$ \\
\hline is obutyl isobutyrate & $\begin{array}{l}\text { intensity abs. } \\
\text { intensity \% } \\
\text { M/F\% } \\
\end{array}$ & n.d. & n.d. & $\begin{array}{r}2291154 \\
100.0\end{array}$ & n.d. & n.d. & n.d. & $\begin{array}{r}2004020 \\
100.0\end{array}$ & n.d. & n.d. & n.d. & $\begin{array}{r}2125342 \\
100.0\end{array}$ & n.d. & n.d. & n.d. & $\begin{array}{r}2085273 \\
100.0 \\
\end{array}$ & n.d. \\
\hline butyl isobutyrate & $\begin{array}{l}\text { intensity abs. } \\
\text { intensity \% } \\
\text { M/F\% }\end{array}$ & n.d. & n.d. & $\begin{array}{r}4357417 \\
100.0\end{array}$ & n.d. & n.d. & n.d. & $\begin{array}{r}4009954 \\
100.0\end{array}$ & n.d. & n.d. & n.d. & $\begin{array}{r}3972002 \\
100.0\end{array}$ & n.d. & n.d. & n.d. & $\begin{array}{r}3935625 \\
100.0\end{array}$ & n.d. \\
\hline 2-methylbutyl 2-methylbutyrate & $\begin{array}{l}\text { intensity abs. } \\
\text { intensity \% } \\
\text { M/F\% } \\
\end{array}$ & n.d. & n.d. & $\begin{array}{r}5710600 \\
100.0\end{array}$ & n.d. & n.d. & n.d. & $\begin{array}{r}4933864 \\
100.0\end{array}$ & n.d. & n.d. & n.d. & $\begin{array}{r}5325653 \\
100.0\end{array}$ & n.d. & n.d. & n.d. & $\begin{array}{r}5202649 \\
100.0\end{array}$ & n.d. \\
\hline 2-methylbutyl 3-methylbutyrate & $\begin{array}{l}\text { intensity abs. } \\
\text { intensity \% } \\
\mathbf{M} / \mathbf{F} \%\end{array}$ & n.d. & n.d. & $\begin{array}{r}5983952 \\
100.0\end{array}$ & n.d. & n.d. & n.d. & $\begin{array}{r}5373223 \\
100.0\end{array}$ & n.d. & n.d. & n.d. & $\begin{array}{r}5605866 \\
100.0\end{array}$ & n.d. & n.d. & n.d. & $\begin{array}{r}5320095 \\
100.0\end{array}$ & n.d. \\
\hline propyl 2-methylbutyrate & $\begin{array}{l}\text { intensity abs. } \\
\text { intensity \% }\end{array}$ & n.d. & $\begin{array}{r}46835 \\
0.5 \\
0.5\end{array}$ & $\begin{array}{r}9790339 \\
100.0\end{array}$ & n.d. & n.d. & $\begin{array}{r}55296 \\
0.6 \\
0.6\end{array}$ & $\begin{array}{r}8921178 \\
100.0\end{array}$ & n.d. & n.d. & $\begin{array}{r}44148 \\
0.5 \\
0.5\end{array}$ & $\begin{array}{r}9222561 \\
100.0\end{array}$ & n.d. & n.d. & $\begin{array}{r}41766 \\
0.5 \\
0.5\end{array}$ & $\begin{array}{r}9126248 \\
100.0\end{array}$ & n.d. \\
\hline & $\frac{M / \%}{\text { intensity abs. }}$ & & 27951205 & 932824 & 46021 & & 29094693 & 951085 & 481111 & & 27436981 & 900038 & 477782 & & 25640735 & 844594 & 41938 \\
\hline methyl 2-methylbutyrate & $\begin{array}{l}\text { intensity \% } \\
\text { M/F\% }\end{array}$ & n.d. & $\begin{array}{r}100.0 \\
2996.4\end{array}$ & 3.3 & 0.2 & n.d. & $\begin{array}{r}100.0 \\
3059.1\end{array}$ & 3.3 & 0.2 & n.d. & $\begin{array}{r}100.0 \\
3048.4\end{array}$ & 3.3 & 0.2 & n.d. & $\begin{array}{r}100.0 \\
3035.9\end{array}$ & 3.3 & 0.2 \\
\hline & intensity abs. & 174988 & 15751490 & 37412428 & & 147135 & 13788566 & 32285308 & & 158233 & 14339113 & 33606071 & & 150133 & 14193298 & 33406345 & \\
\hline methyl geranate & intensity \% & 0.5 & 42.1 & 100.0 & n.d. & 0.5 & 42.7 & 100.0 & n.d. & 0.5 & 42.7 & 100.0 & n.d. & 0.4 & 42.5 & 100.0 & n.d. \\
\hline & & 0.5 & 42.1 & & & 0.5 & 42.7 & & & 0.5 & 42.7 & & & 0.4 & 42.5 & & \\
\hline & intensity abs. & & 103508 & 28035872 & 1726169 & & 86396 & 24474864 & 1455280 & & 91361 & 27145966 & 1694462 & & 89352 & 27027924 & 1744859 \\
\hline geranyl acetate & $\begin{array}{l}\text { intensity \% } \\
\text { M/F\% }\end{array}$ & n... & $\begin{array}{l}0.4 \\
0.4\end{array}$ & 100.0 & & n.d. & $\begin{array}{l}0.4 \\
0.4\end{array}$ & 100.0 & & n.d. & $\begin{array}{l}0.3 \\
0.3\end{array}$ & & 6.2 & n.d. & $\begin{array}{l}0.3 \\
0.3\end{array}$ & & 6.5 \\
\hline & intensity abs. & 9827 & 120208 & 24776478 & 1595497| & & 112400 & 22033526 & 1367854 & 17598 & 117087 & 23688168 & 1426363 & 14874 & 121258 & 23898760 & 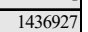 \\
\hline geranyl propionate & intensity \% & 0.0 & 0.5 & 100.0 & 6.4 & n.d. & 0.5 & 100.0 & 6.2 & 0.1 & 0.5 & 100.0 & 6.0 & 0.1 & 0.5 & 100.0 & 6.0 \\
\hline & & 0.0 & 0.5 & & & & 0.5 & & & 0.1 & 0.5 & & & 0.1 & 0.5 & & \\
\hline & nsity abs. & 15255 & 170713 & 13800894 & 1071289 & 6185 & 141912 & 12105756 & 883505 & 12941 & 157924 & 13035879 & 918308 & 13867 & 154436 & 12952596 & 966427 \\
\hline geranyl is obutyrate & intensity \% & 0.1 & 1.2 & 100.0 & 7.8 & 0.1 & 1.2 & 100.0 & 7.3 & 0.1 & 1.2 & 100.0 & 7.0 & 0.1 & 1.2 & 100.0 & 7.5 \\
\hline & M/F\% & 0.1 & 1.2 & & & 0.1 & 1.2 & & & 0.1 & 1.2 & & & 0.1 & 1.2 & & \\
\hline
\end{tabular}

n.d.: ion not detected

F80 and F200: fragment ions at cone gas flows of 80 and $200 \mathrm{~L} / \mathrm{h}$, respectively. 
Table S3. Calculation of the Limit of detection and quantification (LOD, LOQ) for all 22 analytes of the standard mix and mean responses of dilutions of the standard mix.

\begin{tabular}{|c|c|c|c|c|c|c|c|}
\hline analyte & $\mathrm{L}_{\mathrm{B}}^{\mathrm{a}}$ & $\mathrm{S}_{\mathrm{B}}^{\mathrm{b}}$ & $\mathrm{LOD}^{\mathrm{c}}$ & $\mathrm{LOQ}^{\mathrm{d}}$ & $\begin{array}{c}\mathrm{L}_{\mathrm{D}} \\
(10 \mu \mathrm{g} / \mathrm{L})^{\mathrm{e}}\end{array}$ & $\begin{array}{c}\mathrm{L}_{\mathrm{D}} \\
(50 \mu \mathrm{g} / \mathrm{L})^{\mathrm{e}}\end{array}$ & $\begin{array}{c}\text { LOQ } \\
(\mu \mathrm{g} / \mathrm{kg})^{\mathrm{f}}\end{array}$ \\
\hline myrcene & 1205 & 129 & 1593 & 2499 & 9662 & 21828 & 0.150 \\
\hline$(-)-\beta$-pinene & 119 & 49 & 266 & 609 & 4784 & 21050 & 0.150 \\
\hline$(+)$-limonene & 1784 & 210 & 2413 & 3880 & 6835 & 23484 & 0.150 \\
\hline$\alpha$-humulene & 834 & 148 & 1277 & 2310 & 14905 & 72908 & 0.150 \\
\hline$\beta$-caryophyllene & 73 & 25 & 147 & 321 & 11298 & 56389 & 0.150 \\
\hline 2-undecanone & 193 & 60 & 373 & 793 & 7285 & 42649 & 0.150 \\
\hline linalool & 815 & 203 & 1424 & 2847 & 9151 & 30090 & 0.150 \\
\hline$(+)$ - $\alpha$-terpineol & 166 & 107 & 487 & 1238 & 5042 & 24587 & 0.150 \\
\hline cis-linalooloxide & 166 & 46 & 304 & 628 & 7373 & 38206 & 0.150 \\
\hline (-)-caryophyllenoxide & 438 & 81 & 680 & 1245 & 19942 & 99352 & 0.150 \\
\hline 2-methylbutyl isobutyrate & 90 & 23 & 159 & 322 & 734 & 3433 & 0.150 \\
\hline 3-methylbutyl isobutyrate & 94 & 27 & 175 & 366 & 407 & 1825 & 0.150 \\
\hline isobutyl isobutyrate & 194 & 65 & 390 & 847 & 693 & 2647 & 0.750 \\
\hline butyl isobutyrate & 71 & 51 & 224 & 580 & 1378 & 6022 & 0.150 \\
\hline 2-methylbutyl 2-methylbutyrate & 71 & 43 & 201 & 503 & 1829 & 9273 & 0.150 \\
\hline 2-methylbutyl 3-methylbutyrate & 67 & 43 & 196 & 496 & 1377 & 7331 & 0.150 \\
\hline propyl 2-methylbutyrate & 82 & 61 & 264 & 689 & 3670 & 18332 & 0.150 \\
\hline methyl 2-methylbutyrate & 94 & 39 & 210 & 481 & 11381 & 53293 & 0.150 \\
\hline methyl geranate & 70 & 40 & 189 & 468 & 8171 & 47083 & 0.150 \\
\hline geranyl acetate & 117 & 65 & 312 & 769 & 4629 & 27106 & 0.150 \\
\hline geranyl propionate & 116 & 59 & 292 & 703 & 5839 & 34160 & 0.150 \\
\hline geranyl isobutyrate & 186 & 17 & 237 & 357 & 3781 & 16993 & 0.150 \\
\hline \multicolumn{8}{|c|}{ a: mean of the replicate blank responses for 6 -fold determination (target ion see characteristic ion in Table S1) } \\
\hline \multicolumn{8}{|c|}{ b: standard deviation of the replicate blank responses } \\
\hline \multicolumn{8}{|c|}{$\mathrm{c}: \mathrm{LOD}=\mathrm{L}_{\mathrm{B}}+3 * \mathrm{~S}_{\mathrm{B}}$} \\
\hline \multicolumn{8}{|l|}{$\mathrm{d}: \mathrm{LOQ}=\mathrm{L}_{\mathrm{B}}+10 * \mathrm{~S}_{\mathrm{B}}$} \\
\hline
\end{tabular}




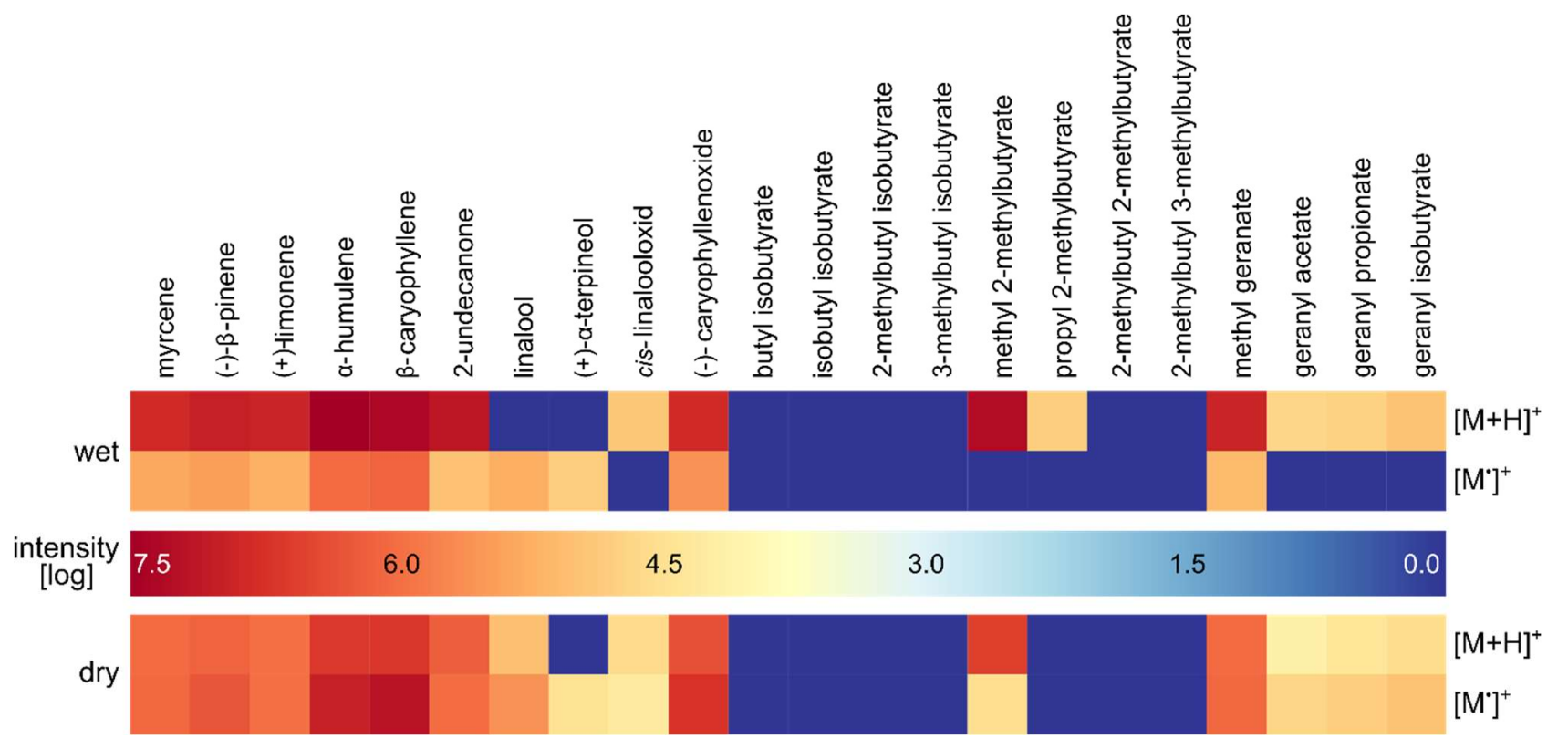

Figure S1. Heatmap of $\log 10$ trans-formed intensities of the molecular ions $\left(\left[\mathrm{M}^{*}\right]^{+}\right)$and protonated molecules $\left([\mathrm{M}+\mathrm{H}]^{+}\right)$for all analytes in the standard mix under dry and wet source conditions. 


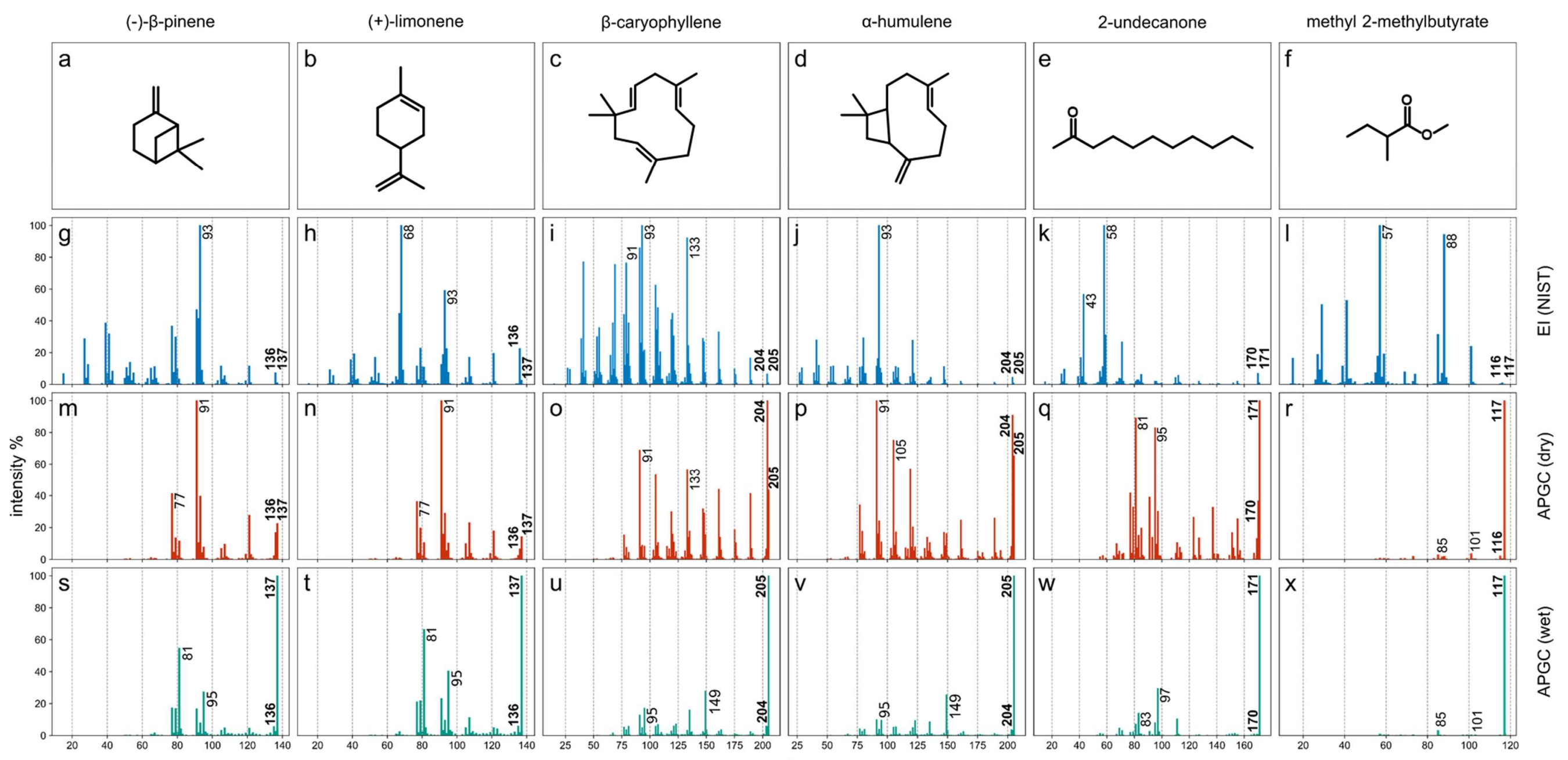

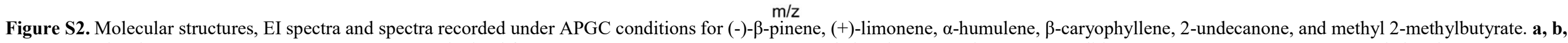

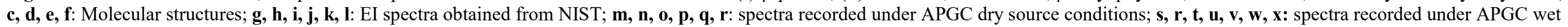

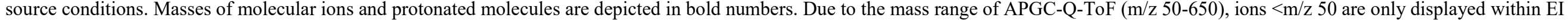
spectra. 


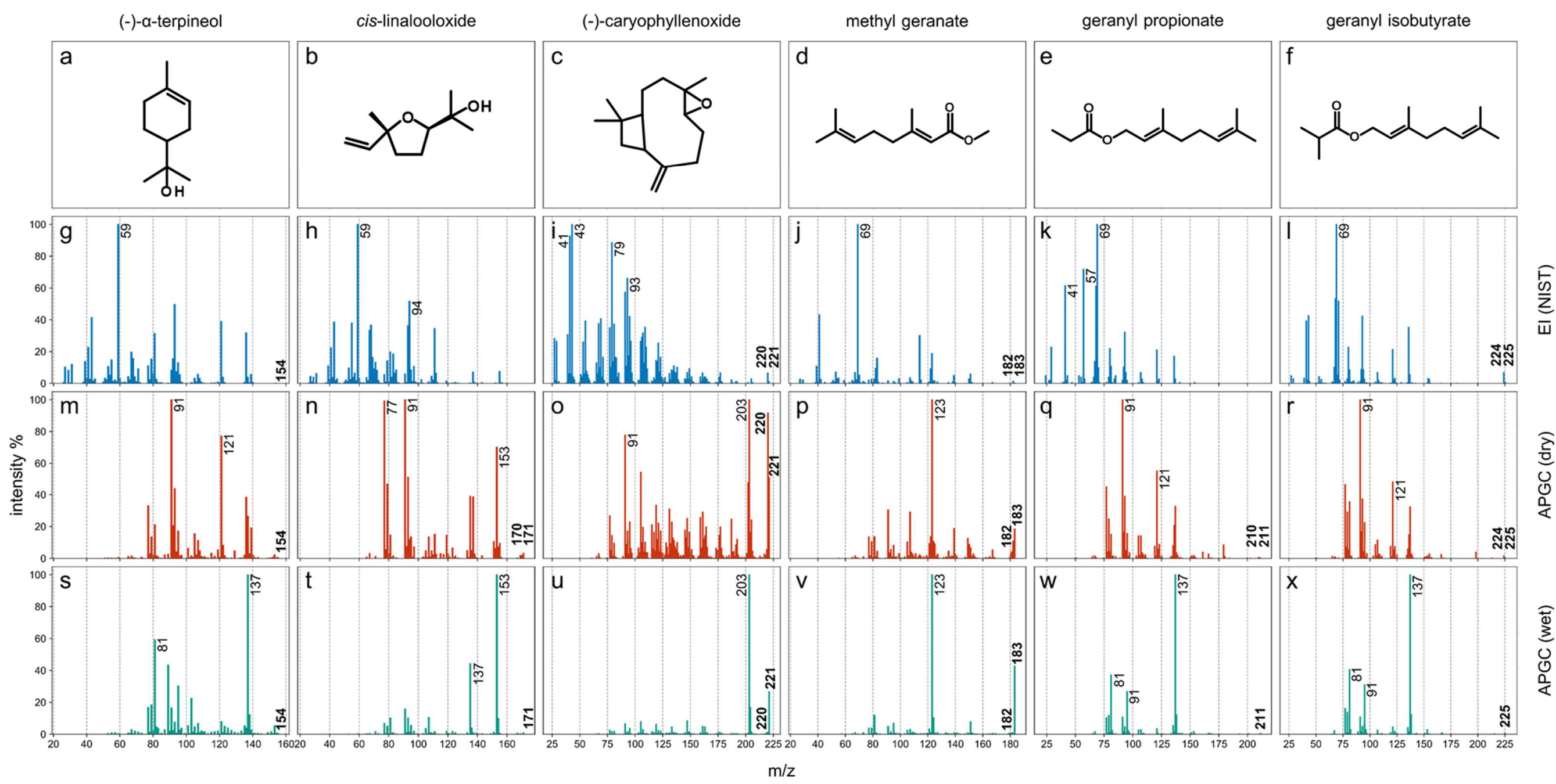

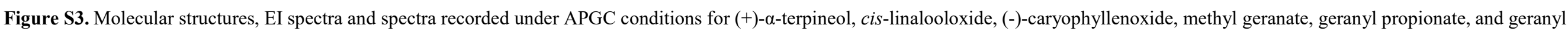

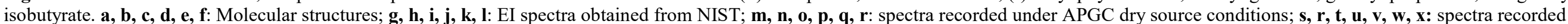

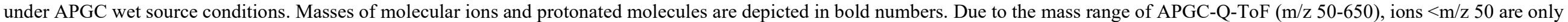
displayed within EI spectra. 


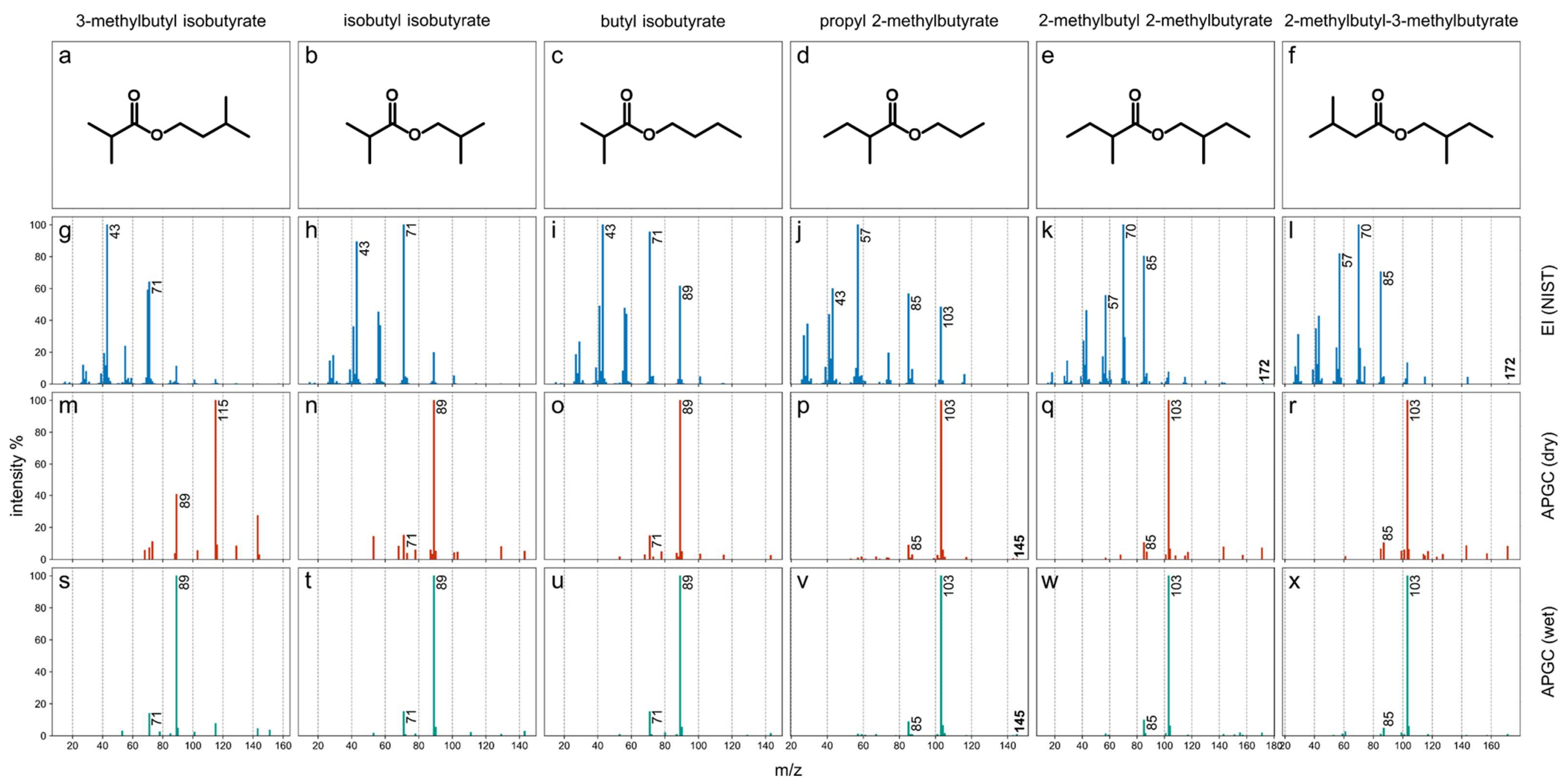

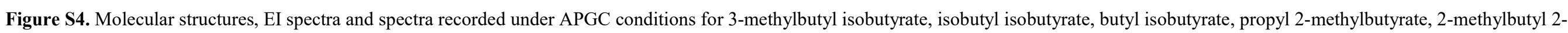

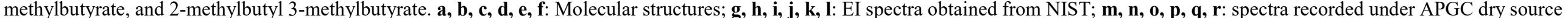

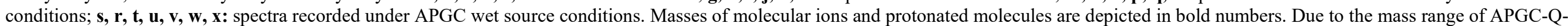
$\mathrm{ToF}(\mathrm{m} / \mathrm{z} 50-650)$, ions $<\mathrm{m} / \mathrm{z} 50$ are only displayed within EI spectra. 

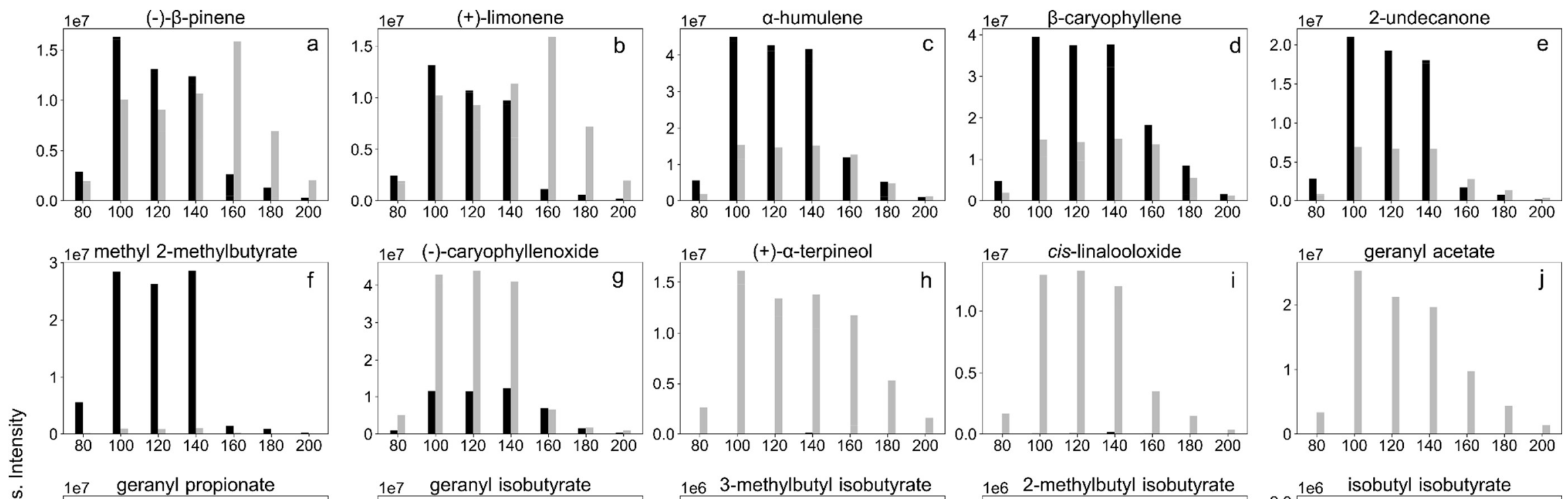

\author{
$1 \mathrm{e} 7 \quad(+)$-a-terpineol
}
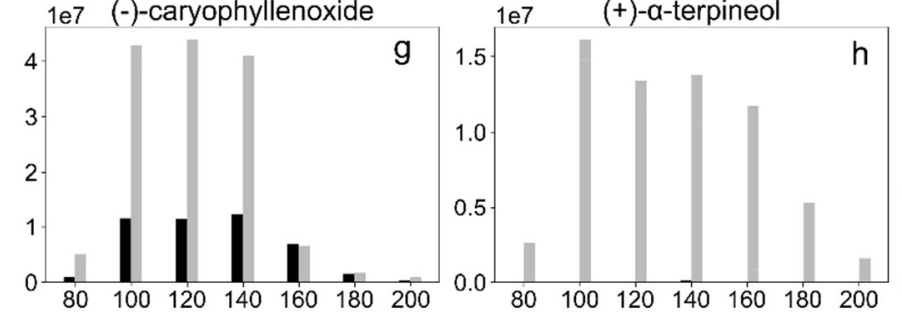

1e7 cis-linalooloxide

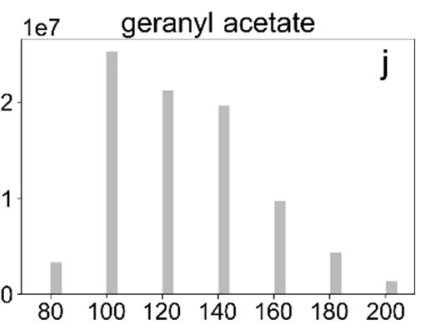

宁 $2 . 0 \longdiv { \mathrm { e } 7 }$ geranyl propionate
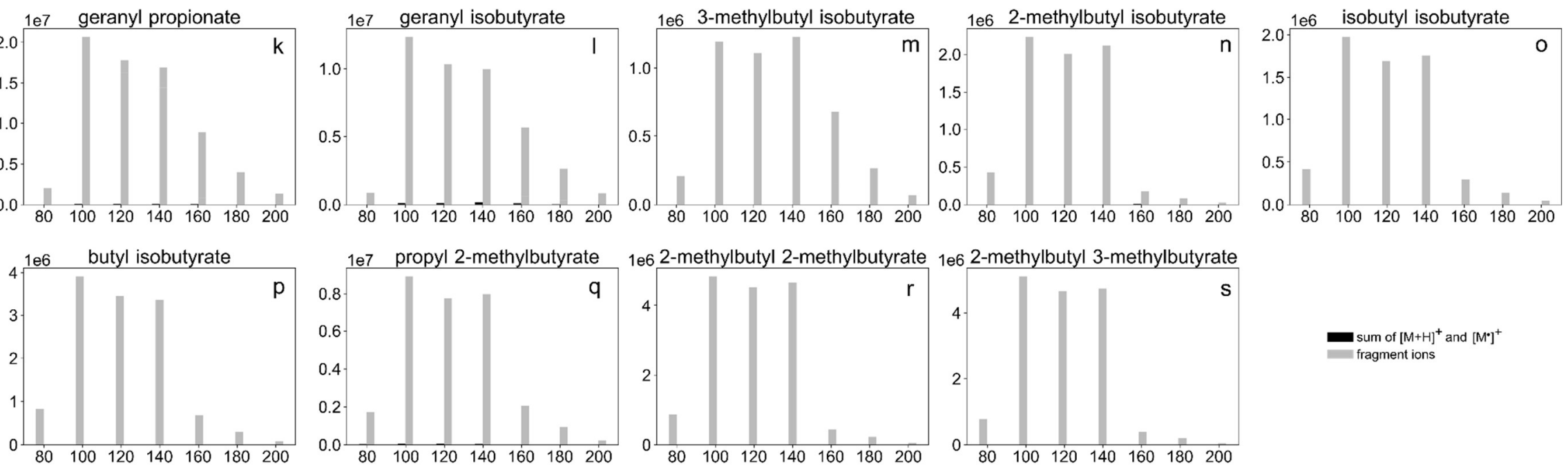

$80 \quad 100120140160 \quad 180200$

cone gas flow $\mathrm{L} / \mathrm{h}$

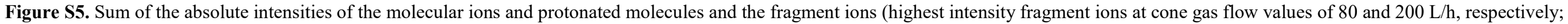

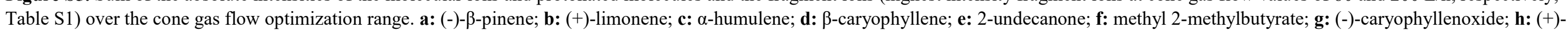

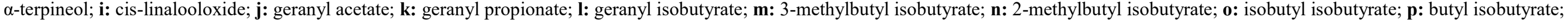
q: propyl 2-methylbutyrate; r: 2-methylbutyl 2-methylbutyrate; s: 2-methylbutyl 3-methylbutyrate. 


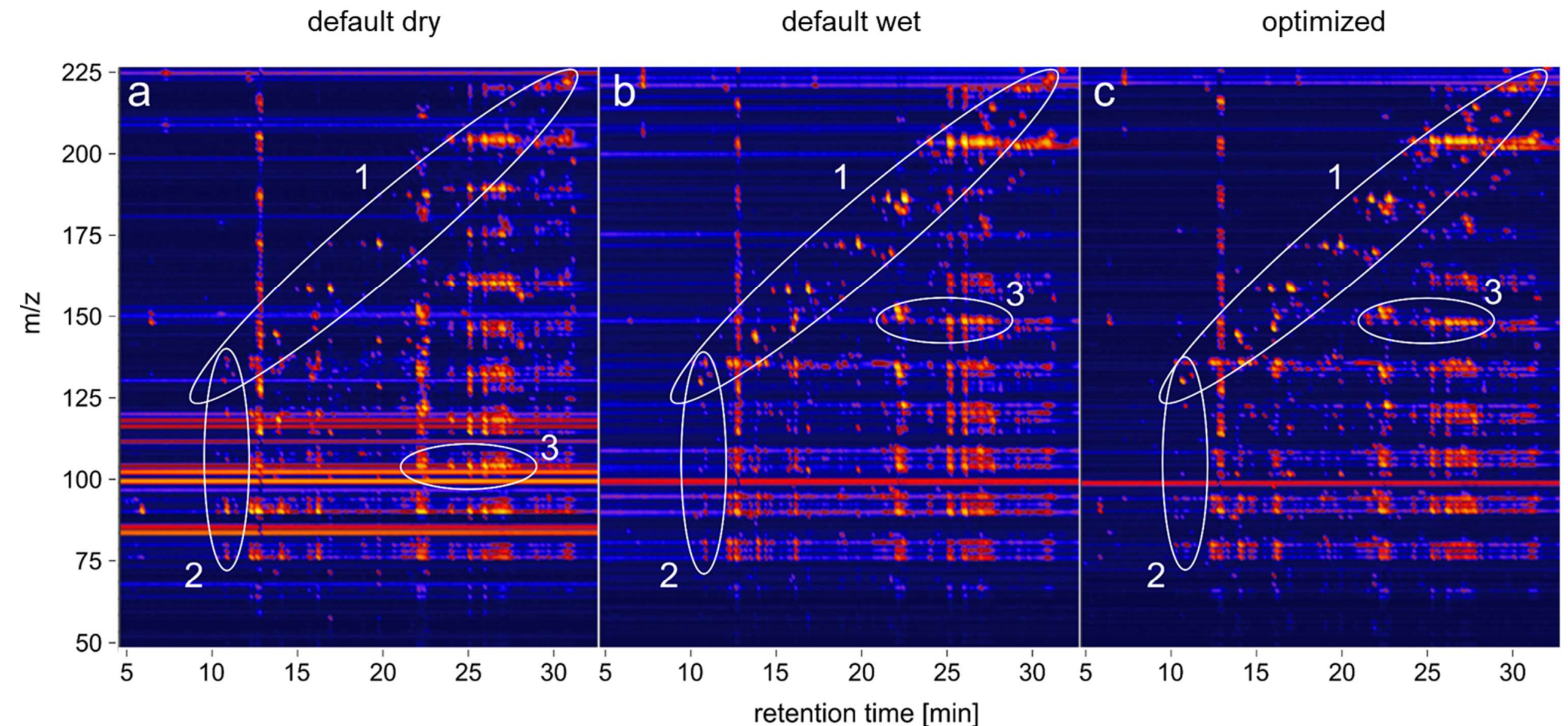

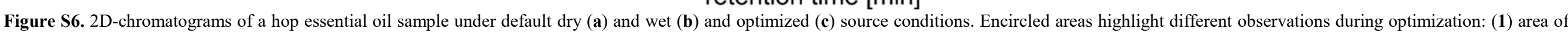
potential molecule ions; (2) proportion of fragment ions to molecule ion; (3) fragment with the highest intensity. 